\title{
Cluster Preface: Synthesis of Materials
}

\author{
Timothy M. Swager* \\ Department of Chemistry, Massachusetts Institute of Technology, \\ 77 Massachusetts Avenue, Cambridge, MA 02139, USA \\ tswager@mit.edu
}

Received: 14.10.2018

Accepted: 14.10 .2018

Published online: 21.11 .2018

DOI: 10.1055/s-0037-1610835; Art ID: st-2018-s5000-c

Timothy M. Swager is the John D. MacArthur Professor of Chemistry and the Director, Deshpande Center for Technological Innovation at the Massachusetts Institute of Technology. A native of Montana, he received a BS from Montana State University in 1983 and a Ph.D. from the California Institute of Technology in 1988. After a postdoctoral appointment at MIT he was on the chemistry faculty at the University of Pennsylvania and returned to MIT in 1996 as a Professor of Chemistry and served as the Head of Chemistry from 2005 to 2010. He has published more than $450+$ peer-reviewed papers and more than $90+$ issued/pending patents. Swager's honors include: Election to the National Academy of Sciences, an Honorary Doctorate from Montana State University, the Linus Pauling Medal, the Lemelson-MIT Award for Invention and Innovation, Election to the American Academy of Arts and Sciences, The American Chemical Society Award for Creative Invention, and The Carl S. Marvel Creative Polymer Chemistry Award (ACS). Swager's research interests are in design, synthesis, and study of organicbased electronic, sensory, high-strength, liquid crystalline, and colloid materials. His inventions have had wide-ranging commercial impact, including the Fido ${ }^{\mathrm{TM}}$ sensors, which are the world's most sensitive explosives detectors. He is the scientific founder of five companies (DyNuPol, Iptyx, Polyjoule, $C_{2}$ Sense, and Xibus Systems) and has served on numerous corporate and government boards.

\section{Efficient Synthesis + Extraordinary Molecules = Future Advanced Materials}

Although there is still the occasional new inorganic material composition constructed from combining atomistic components, the vast majority of new emerging materials are built from the bottom-up by the connection and assembly of molecules. Chemistry has produced a dazzling array of synthetic methods, spectroscopies, and theoretical understanding that can be broadly practiced to create materials with complex geometric structures, electronic charac-

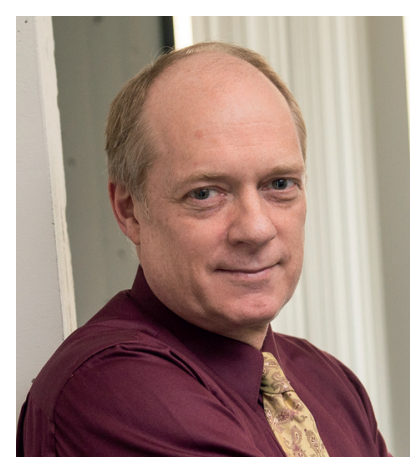

teristics, optical properties, and chemical potentials. It is tempting to compare the evolution of the synthetic materials world to that of nature, wherein molecular subunits such as amino acids, nucleotide bases, and carbohydrates are connected and arranged, often with the assistance of inorganic ions and water, into functional assemblies. Although nature's amazing evolutionary accomplishments defy comprehension, chemists have already shown the power of applying a much more diverse set of building blocks to create extraordinary materials. Aromatic amides, for example, give hydrogen bonding networks and mechanical strength in the material Kevlar ${ }^{\mathrm{TM}}$, which exceeds all natural proteins. However, we are still struggling to create materials that translate the properties of microscopic spider silk fibers to bulk systems. Some systems are simply tough to scale. Perhaps a clever chemist will develop the proper molecules with the proper balance of mechanics and conformational complexity to effect this transition.

The chemistry toolbox is full of important opportunities for materials creation. Anisotropic molecules can be made to assemble in predictable ways. Carefully tailored intermolecular potentials can be used similar to the base-pairing in nucleotides, but need not be limited to hydrogen bonding and can include halogen bonding, metal-ligand associations, charge transfer, $\pi$-stacking, and host-guest complexation. Chemistry has an armada of systems with designed reactivity and structural changes that can be powered (actuated) chemically, electrochemically, thermally, or by light, that are certain to create new generations of autonomous self-regulating systems. The unraveling of complex biomolecular reaction networks with feedback mechanisms, agonists and antagonists, is presenting chemistry with many grand challenges. 
We need not limit our inspiration for the design of synthetic materials to nature. Indeed, microelectronic and mechanical systems have evolved to our advantage by continually creating complexity and miniaturization. Moreover, much of our fascination with electronically and optically active molecular materials is inspired by the diodes, photocells, transistors, logic gates, and memory elements created in modern semiconductor electronics.

I am well aware that writing flowery prose about these inspirational connects is the easy part. It is the creation and manipulation of the molecular components where the heavy lifting is needed. Hence, this cluster is focused on leading researchers creating molecular constructions that are building brick by brick a foundation for the construction of new generations of materials. Clearly this is going to be a long, and perhaps never-ending process, but refining our methods to create new molecular properties and structures with ever-improving efficiency, is necessary to empower future materials. The need for precision in synthesis cannot be overstated and defects often limit a materials performance. Hence, the mission of Synlett is very much aligned to highlight important new methods for the creation of molecules that can, in turn, enable the generation of transformative new materials. I hope you enjoy this cluster and the numerous chemical innovations reported. As synthetic chemists, we begin with small things, but ultimately these constituents and methods will enable new integrated materials systems. 